\title{
Children with First Episode of Fever with Seizure: Is Lumbar Puncture Necessary?
}

\author{
Joshi Batajoo R, ${ }^{1}$ Rayamajhi A, ${ }^{1}$ Mahaseth $C^{1}$ \\ 'Kanti Children's Hospital, Kathmandu, Nepal
}

\section{ABSTRACT}

Fever with seizure, a common presentation with which a child may present to the emergency is mainly due to febrile seizure, but it may also be due to meningitis. This study was done to find out the incidence of meningitis and to find out whether lumbar puncture is necessary in different age groups of children presenting with first episode of fever with seizure.

A prospective study was conducted in the emergency department of Kanti Children's Hospital. Children who presented with first episode of fever and seizure in the age group of 6 months to 5 years were included. Meningitis was diagnosed on the basis of either cytological and biochemical criteria or if a bacterial pathogen was isolated.

Of the 175 children included, 17\% were diagnosed to have meningitis. Cerebrospinal fluid was positive for a bacterial pathogen in $4.5 \%$ of the cases. In the age group of 6 months to 12 months, 30\% of the children had meningitis as compared to $20 \%$ and $5 \%$ in other age groups of 12 - 18 months and above 18 months respectively. All children with culture proven bacterial meningitis were in the age group of 6-12 months and had no evidence of meningeal irritation. Signs of meningeal irritation had high specificity in diagnosing meningitis. Organisms grown were Haemophilus influenza in three cases, Streptococcus pneumoniae in two cases and Staphylococcus aureus in three cases.

In conclusion, incidence of meningitis was found to be high in children presenting with first episode of fever and seizure. Lumbar puncture to rule out meningitis should especially be considered in children in the younger age group even without evidence of meningeal irritation.

Key words: cerebrospinal fluid, febrile seizure, lumbar puncture, meningitis

\section{INTRODUCTION}

Fever with seizure is the most common type of seizure occurring in children. ${ }^{1}$ This may be due to febrile seizure or a more ominous condition like meningitis. Seizure is a common presentation for which a child may come to the emergency and may occur in up to $10 \%$ of children presenting to the emergency. ${ }^{2}$ Probability of bacterial meningitis in children with fever with seizure varies from $0.6 \%$ to $6.7 \% .^{3}$ Therefore it is important to differentiate benign febrile seizure from meningitis early and implement appropriate management. 
Joshi Batajoo et al. Children with First Episode of Fever with Seizure: Is Lumbar Puncture Necessary?

Deciding whether a child has meningitis will be easier in older children where there may be meningism or photophobia. In younger children, it may be more difficult to diagnose meningitis and should be considered where there is a history of irritability, decreased feeding, lethargy, prolonged seizure, focal or multiple seizures, prolonged postictal altered consciousness, neurological deficit and prior intake of antibiotics. ${ }^{4}$

According to the American Academy of Pediatrics (AAP) guideline, all children with their first febrile seizure under the age of 12 months should have a lumber puncture (LP) since the signs of meningitis are subtle and also potential risks of delayed treatment of meningitis outweighs any risk of performance of LP. In the age group of 12 to 18 months as it is debatable whether meningitis can be diagnosed solely on the basis of clinical features, LP should be strongly considered. In the age group above 18 months, clinical features of meningeal irritation are usually present; therefore, decision for LP should be done depending on clinical examination. ${ }^{5}$ Different studies have found the incidence of meningitis in first episode of fever with seizure to be different but varying in range from 0.6- $6.7 \% .^{6-13}$

This study was conducted with the objectives to find out the incidence of meningitis and therefore, to determine whether LP is necessary in children aged 6 months to five years presenting with first episode fever with seizure.

\section{MATERIAL AND METHODS}

The study was a hospital based prospective study, conducted in the department of Emergency of Kanti Children's Hospital. Data was collected from August 2004 to January 2005. The study population was 175 children aged six months to five years who presented with first episode of fever with seizure. Children with other neurological disease like cerebral palsy, mental retardation; fever after occurrence of seizure or on antibiotic for more than 48 hours were excluded from the study.

Children were considered as having fever if axillary temperature recorded at the emergency was $>100.4^{\circ}$ F. All patients were examined in the emergency by Pediatric post-graduate students posted in Kanti Children's Hospital as per standard examination methods to minimize inter observer error in detection of meningeal signs. Lumbar puncture was performed and CSF was collected in 2 sterile vials in all enrolled patients. One vial was sent for cell count, differential count, sugar and protein and the other for Gram's stain and culture. The CSF was analyzed as per standard laboratory methods by laboratory technicians.

Meningitis was diagnosed in a child presenting with fever and seizure if he/she had a combination of all three of the following: CSF cells $>5 / \mathrm{mm}^{3}$, protein more than
$40 \mathrm{mg} \%$ and sugar $<2 / 3$ of blood sugar. ${ }^{14}$ Growth of bacteria in the CSF and/or positive Gram's stain was considered as bacterial meningitis. Those growing contaminated organisms were excluded in the analysis. Informed verbal and written consent was taken from the parents or the accompanying adults before enrollment into the study.

\section{RESULTS}

A total of 175 cases that presented with first episode of fever and seizure between six months to five years of age were included. Among them, $121(69.1 \%)$ were male and $54(30.9 \%)$ were female children. There were a total of 53 children in 6-12 months age, 43 in 12-18 months and 79 in above 18 months age group (Figure 1).

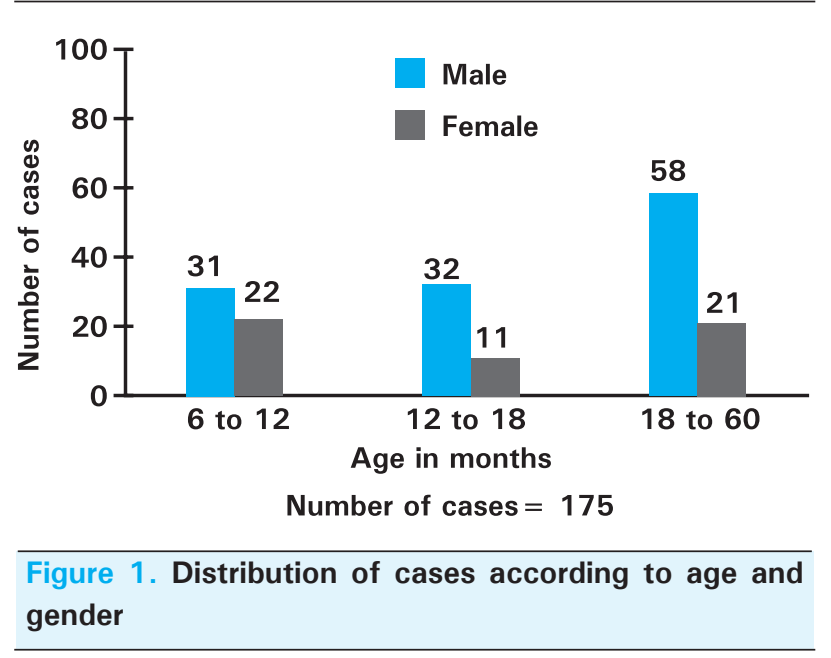

Among the total cases, 30 cases $(17.1 \%)$ had meningitis and 145 cases had febrile seizures. Out of the meningitis cases, 8 cases $(4.5 \%)$ had culture positive bacterial meningitis (Figure 2).

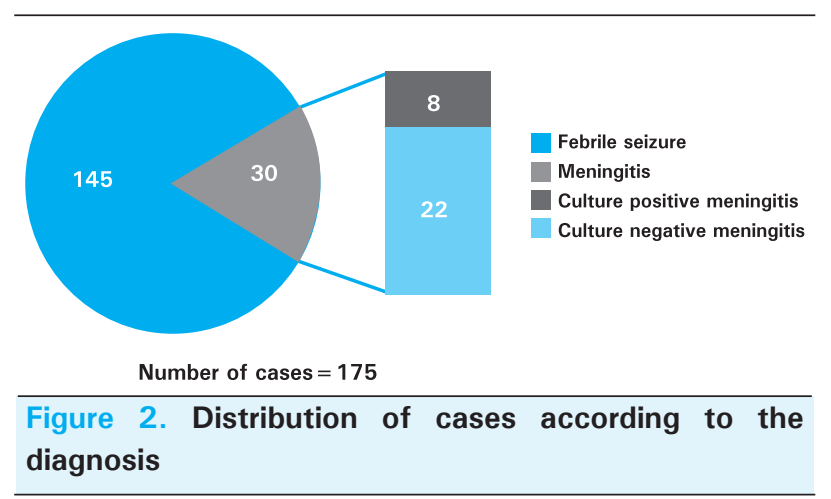

Children of less than 12 months were found to have meningitis significantly ( $p=0.001)$ as compared to older children. In the age group of $6-12$ months, 17 cases $(32 \%)$ had meningitis while in $12-18$ months, 9 out of 43 cases $(20 \%)$ had meningitis and in more than 18 months age group 4 out of 79 cases $(5 \%)$ were detected with meningitis (Table 1). 
Joshi Batajoo et al. Children with First Episode of Fever with Seizure: Is Lumbar Puncture Necessary?

Positive meningeal signs were significantly $(p=$ 0.00002 ) associated with the occurrence of meningitis and it was seen to have a low sensitivity but a high specificity for diagnosing meningitis (Table 2). A significant $(p=0.00008)$ number of children between 6 to 12 months of age had meningitis without signs of meningeal irritation as compared to older children (Table 3). In 8 cases, a bacterial pathogen was grown. Three grew Haemophilus influenza, two grew Streptococcus pneumoniae and three grew Staphylococcus aureus. All culture positive meningitis cases were in the age group of 6 months to 12 months.

Table 1. Distribution of meningitis in different age groups

\begin{tabular}{lccc}
\hline Age in months & Total & \multicolumn{3}{c}{ Meningitis } \\
\hline $6-12$ & 53 & 17 & $(32.1 \%)$ \\
$12-24$ & 62 & 11 & $(17.7 \%)$ \\
$24-36$ & 32 & & 0 \\
$36-48$ & 13 & & 0 \\
$48-60$ & 15 & 2 & $(13.2 \%)$ \\
Total & 175 & 30 & $(17.1 \%)$ \\
\hline
\end{tabular}

$P$ value $=0.001$

Table 2. Meningitis as predicted from meningeal signs

\begin{tabular}{lll}
\hline Meningeal signs & Meningitis & No meningitis \\
\hline Present & 6 (a) & 4 (b) \\
Absent & 24 (c) & $141(\mathrm{~d})$ \\
Total & 30 & 145
\end{tabular}

Using a, b, c, d, Sensitivity $=20 \%$, Specificity $=97 \%$, Positive predictive value $=60$, Negative predictive value $=85$, Odds ratio $=8.8, P$ value $=0.00002$.

\section{DISCUSSION}

Meningitis is a medical emergency in children and should not be missed in any children with fever with seizure. Among the 175 cases, 30 had meningitis showing that almost one fifth of children presenting in the emergency room with apparent febrile seizure have meningitis. Eight cases had culture positive meningitis while 22 cases had meningitis based on CSF cytological and biochemical criteria.
In another study done in $\mathrm{KCH}, 10.9 \%$ of patients with apparent first febrile seizure had meningitis. ${ }^{15}$ Other studies on meningitis in apparent febrile seizure have found the incidence of meningitis to vary from $2-7 \% .^{7-}$ $11,13,16$ Our finding was comparatively higher than from studies done outside the country. The culture yield of CSF was $4.5 \%$, which is comparable to other studies of febrile seizure. ${ }^{9,10,12,15}$ From the high incidence of meningitis detected, it can be said that meningitis should be considered as a provisional diagnosis in all children presenting with fever with seizure.

The younger the patient, the more was the risk of the meningitis. In 6-12 months age group, 32\% had meningitis as compared to $20 \%$ in $12-18$ months age group and $5 \%$ in more than 18 months age group. Other studies have also come to the same conclusion. ${ }^{10-13}$ Therefore, younger the age more is the chance that a child presenting with fever and seizure may have meningitis.

Meningeal signs were found to be significantly associated with meningitis with a specificity of $98 \%$ and negative predictive value of $85 \%$. The high risk of meningitis in the presence of meningeal sign in a patient with fever and seizure is found to be comparable to other studies. ${ }^{7,11}$

It was seen that in the younger age group, there was significant probability of having meningitis without signs of meningeal irritation than in older children. Twenty-four children altogether had meningitis without meningeal sign. Around thirty percent of children in the age group of 6-12 months had meningitis without meningeal signs as compared to $17.1 \%$ in more than $12-18$ months of age and $2.7 \%$ in more than 18 months age group. This is comparable to other studies. ${ }^{8,11}$ All eight cases of culture positive meningitis in this study were in the age group of 6-12 months of age and none of them had meningeal signs.

\section{CONCLUSION}

In conclusion, it was found that meningitis is a common presentation in the emergency and in cases of apparent febrile seizure, meningitis should always be considered as a differential diagnosis. Lumbar puncture is necessary to rule out meningitis in all children between the ages of 6 months to 12 months presenting with first episode of fever with seizure to rule out meningitis, even in the absence of meningeal signs.

Table 3. Meningitis in different age groups with/without meningeal signs

\begin{tabular}{llclc}
\hline \multirow{2}{*}{ Age (months) } & \multicolumn{2}{c}{ With meningeal signs } & \multicolumn{2}{c}{ Without meningeal signs } \\
& Meningitis & No meningitis & Meningitis & No meningitis \\
\hline $6-12$ & $2(50 \%)$ & $2(50 \%)$ & $15(30.6 \%)$ & $34(69.4 \%)$ \\
$>12-18$ & $2(100 \%)$ & 0 & $7(17.1 \%)$ & $34(82.9 \%)$ \\
$>18-60$ & $2(50 \%)$ & $2(50 \%)$ & $2(2.7 \%)$ & $73(77.3 \%)$ \\
$P$ value & & 0.435 & & 0.00008 \\
\hline
\end{tabular}




\section{REFERENCE}

1. Chin RFM, Neville BGR, Scott RC. Meningitis is a common cause of convulsive status epilepticus with fever. Arch Dis Child 2005;90:66-69.

2. Johnston MV. Seizures in childhood. In: Behrman RE, Kliegman RM, Jenson HB, editors. Nelson textbook of pediatrics.17 ${ }^{\text {th }}$ ed.Saunders press;2004. p. 1993-1994.

3. Armon K, Stephenson T, MacFaul R, Hemingway P, Werneke $\mathrm{U}, \mathrm{Smith} \mathrm{S}$. An evidence and consensus based guideline for the management of a child after a seizure. Emerg Med J 2003;20:13-20.

4. Waruiru C, Appleton R. Febrile seizures: an update. Arch Dis Child 2004;89: 751-756.

5. Provisional Committee on Quality Improvement, Subcommittee on Febrile Seizures Practice Parameter. The neurodiagnostic evaluation of the child with a first simple febrile seizure. Pediatrics 1996;97:5: 769-771.

6. Trainor JL, Hampers LC, Krug SE, Listernick R. Children with first-time simple febrile seizures are at low risk of serious bacterial illness. Acad Emerg Med 2001;8: 781-787.

7. Akpede GO, Sykes RM, Abiodun PO. Indication for lumbar puncture in children presenting with convulsions and fever of acute onset: experience in the children's emergency room of the University of Benin Teaching Hospital, Nigeria. Ann Trop Paediatr 1992;12:4: 385-9.

8. Lorber J, Sunderland R. Lumbar puncture in children with convulsions associated with fever. Lancet 1980 Apr 12;1:8172: 785-6.
9. Joffe A, McCormick M, DeAngelis C. Which children with febrile seizures need lumbar puncture? A decision analysis approach. Am J Dis Child 1983 Dec;137: 1153-6.

10. AkpedeGO, Sykes RM. Convulsions with fever as a presenting feature of bacterial meningitis among preschool children in developing countries. Dev Med Child Neurol 1992 Jun;34:6: 524-9.

11. Jaffe M, Bar-Joseph G, Tirosh E. Fever and convulsions indications for laboratory investigations. Paediatrics 1981;67: 729-31.

12. Laditan AA. Analysis of the results of routine lumbar puncture after a first febrile convulsion in Hofuf, Al- Hassa, Saudi Arabia. East Afr Med J 1995 June: 376-8.

13. Abuekteish F, Daoud AS, al-sheyyab M, Nou'man M. Demographic characteristics and risk factors of first febrile seizures: a Jordanian experience. Trop Doct 2000 Jan;30(1): $25-7$.

14. Haslam RHA. Neurological evaluation. In: Behrman RE, Kliegman RM, Jenson HB, editors. Nelson textbook of pediatrics.17 $7^{\text {th }}$ ed.Saunders press;2004. p. 1980-1981

15. Belbase RH. Demographic profile and CSF findings in first episode of febrile seizure (MD thesis). Kathmandu: Institute of Medicine; 2004.

16. Teach SJ, Geil PA. Incidence of bacteremia, urinary tract infections, and unsuspected bacterial meningitis in children with febrile seizures. Pediatr Emerg Care 1999 Feb;15(1):912. 